\title{
THREE-DIMENSIONAL ASSEMBLY SYNTHESIS FOR ROBUST DIMENSIONAL INTEGRITY BASED ON SCREW THEORY
}

\author{
Byungwoo Lee \\ Department of Mechanical Engineering \\ University of Michigan \\ USA \\ byungwoo@umich.edu \\ Kazuhiro Saitou \\ Department of Mechanical Engineering \\ University of Michigan \\ USA \\ kazu@umich.edu
}

\begin{abstract}
This paper presents a $3 D$ extension of our previous work on the synthesis of assemblies whose dimensional integrity is insensitive to the dimensional variations of individual parts. Assuming that assemblies can be built in the reverse sequence of decomposition, the method recursively decomposes a given product geometry into two subassemblies until parts become manufacturable. At each recursion, joints are assigned to the interfaces between two subassemblies to ensure the two criteria for robust dimensional integrity, in-process dimensional adjustability and proper part constraints. Screw Theory is utilized as a unified $3 D$ representation of the two criteria. A case study on an automotive space frame is presented to demonstrate the method.
\end{abstract}

\section{KEYWORDS}

Design for assembly, design optimization, computeraided design, assembly synthesis.

\section{INTRODUCTION}

Structural enclosures of modern mechanical products, such as ship hulls, airplanes and automotive bodies, typically are made of hundreds or thousands of parts due to their geometric complexity and sizes. As the number of parts increases, however, achieving the dimensional integrity of the final assembly becomes more difficult due to the inherent variations in manufacturing and assembly operations.

A solution is to adjust critical dimensions during an assembly when parts are located and fully constrained in fixtures. This in-process dimensional adjustment is typically facilitated by slip planes, mating surfaces at joints that allow a small amount of relative motions. For example, Figure 1 shows two designs of a rectangular box. In contrast to design in (a) with no in-process adjustability of the critical dimensions (length between sections 1 and 3), design in (b) provides slip planes such that relative location of parts can be adjusted along the critical dimension. (a)

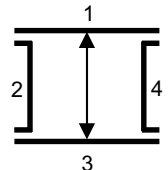

(b)

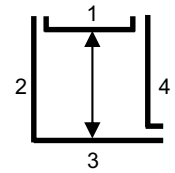

Figure 1 Two box designs (a) without and (b) with adjustable height during assembly (Lee and Saitou, 2003a).

The dimensional integrity of an assembly is also affected by the post-assembly distortion due to the internal stress induced by joining parts with dimensional mismatches. A solution is to ensure a proper constraint of parts at each assembly step. For example, part 1 in Figure 2 (a) is not properly constrained and therefore the post-assembly distortion might occur, if the length of sections 2 and 4 are slightly different due to manufacturing variation. With two slip planes perpendicular to each other, the design in (b) can absorb manufacturing variations within section 1 and 2-3-4, provided that variations in angles are negligible.

In addition to the decomposition of product geometry and the assignment of joint types at part interfaces, the assembly sequence also influences the in-process (a)

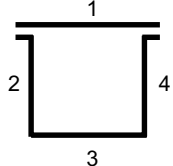

(b)

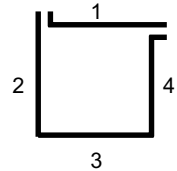

Figure 2 Two box designs (a) without and with (b) proper constraints (Lee and Saitou, 2003a). 
dimensional adjustability and proper part constraints. In an assembly sequence in Figure 3 (a), the critical dimension (total length) is not adjustable since there is no slip plane when the total length is determined with the addition of part 1 . On the other hand, the sequence shown in (b) provides the slip plane at the assembly step where the critical dimension is achieved, to absorb a variation in length. As another example, the sequence in Figure 4 (b), where each critical dimension is independently adjusted at each step, is more desirable than the sequence in (a), where both dimensions are adjusted by one step, inevitably requiring a compromise between two critical dimensions.

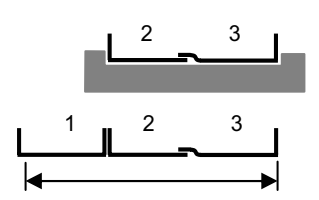

(a)

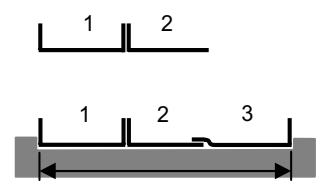

(b)
Figure 3 Assembly sequences (a) without and (b) with in-process adjustability (modified from Whitney, et al., 1999).

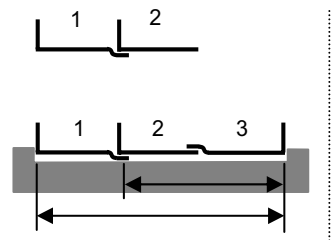

(a)

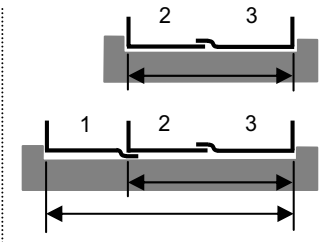

(b)
Figure 4 Assembly sequences where two dimensions are adjusted (a) at one step and (b) independently at two steps (modified from Whitney et al., 1999).

Figure 5 illustrates an effect of the assembly sequence on proper part constraints, where the sequence in (a) causes improper constraint of part 1 at the second step, whereas all parts are properly constrained at all steps in the sequence in (b).

As illustrated so far, the in-process adjustability and proper part constraints are effective tools for achieving high dimensional integrity of an assembly without requiring high part tolerances (Blanding, 1999). The use of these tools in complex assemblies can be a very tedious task due to the coupling between the product decomposition, joint assignments, and assembly sequences. As a remedy, we have previously designed (Lee and Saitou, 2003a) a correct and complete algorithm to fully enumerate feasible solutions for any 2D enclosure geometry. Assuming that assemblies can be built in the reverse sequence of decomposition, the algorithm recursively decomposes a given product geometry into two subassemblies until parts become manufacturable. At each recursion, joints are assigned to the interfaces between two subassemblies to ensure in-process dimensional adjustability and proper part constraints. (a)
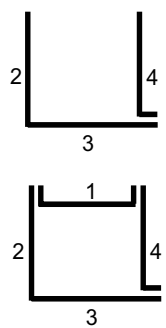

(b)
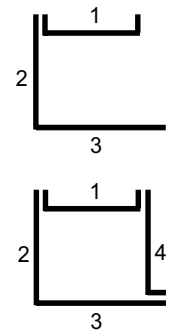

Figure 5 Assembly sequences (a) without and (b) with proper constraints (Lee and Saitou, 2003a).

This paper presents a 3D extension of the algorithm, where Screw Theory (Ball, 1900) is utilized as a unified 3D representation of in-process adjustability and proper part constraints. Dissimilar to our previous work that assumes joints with arbitrary mating angles, they are selected from a library of feasible joints specific to the application domain. A case study on an automotive space frame is presented to demonstrate the method.

\section{RELATED WORKS}

Previous works related to assembly synthesis in general are reviewed in (Lee and Saitou, 2003a). Due to the space limit, this section focuses on the works relevant to the $3 \mathrm{D}$ extension of the method.

The advantages of properly constrained assemblies are well known to practitioners in precision machinery design and several methods have been proposed in literatures including: Kinematic Design (Whitehead, 1954), Minimum Constraint Design (Kamm, 1990) and Exact Constraint Design (Kriegel, 1995; Blanding, 1999). These works describe disadvantages of over-constraint through examples and provide good practices as well as analytical methods to compute constraints. In these works, the most commonly cited merit of properly constraint design is repeatability which leads to high precision. Recently, there has been a trial to analyze and classify key features that enables properly constraint design (Downey, et al., 2002). 
A universal analytical method for motion and constraint analysis dates back to Screw Theory, a pioneering work by Ball (1900). Since then, Screw Theory has been applied to areas of mechanism, robotics and machine design. Among others, Waldron (1964) utilized the screw theory to build a general method which can determine all relative degrees of freedom (DOF) between any two rigid bodies making contacts to each other. Recently, Konkar and Cutkosky (1995) has proposed a recursive algorithm which computes motions allowed by mating features within mechanisms. Adams and Whitney (1999) have extended this method by providing a dual method to compute the state of constraint of parts and applied it to rigid body assemblies with mating features such as pin-slot joint

While these works provide tools for analyzing constraints in a given assembly and simple design guidelines, they do not address a systematic synthesis of an assembly with desired constraint characteristics such as in-process dimensional adjustability and proper part constraints, as discussed in this paper.

\section{TERMINOLOGY}

Since the assembly synthesis deals with objects yet to be decomposed into an assembly of separate parts, a few terms need to be defined to avoid confusion with generic meanings used in other literatures.

- A product geometry is a geometric representation of a whole product as one piece (Figure 6 (a)).

- A member is a section of a product geometry allowed to be a separate part. A pair of members is connected when they meet at a certain point in the product geometry.

- A configuration is a group of members which are connected to at least one member within the group. A product geometry is a configuration, so as a part (as defined below).

- The Key Characteristics (KCs) are defined by Lee and Thornton (1996) as product features, manufacturing process parameters, and assembly features that significantly affect a product's performance, function and form. In this paper, $\mathrm{KC}$ refers to a critical dimension to be achieved in assemblies.

- A configuration graph (or simply configuration if unambiguous in the context) is a triple

$$
C=(M, E, A)
$$

\footnotetext{
* Previously defined in Lee and Saitou (2003b).
}

where $M, E$, and $A$ are the sets of nodes representing members, edges representing connections between two members, and edges representing KCs, respectively (Figure 6 (b)).

- A decomposition is a transition of a configuration into two or more subconfigurations by removing connections between two members.

- A part is a configuration that is not decomposed further under given criteria, eg., a minimum part size. A part may consist of one or more members.

- A joint library is a set of joint types available for a specific application domain (Figure 7).

- An (synthesized) assembly is a set of parts and joints that connect every part in the set to at least one of other parts in the set.

- Assembly synthesis is a transformation of a product geometry into an assembly. (a)

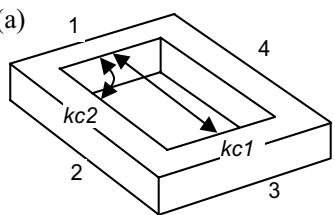

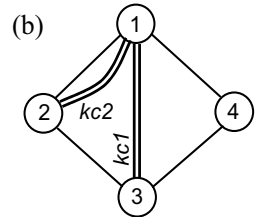

Figure 6 (a) product geometry of a beam based product and (b) its configuration graph.
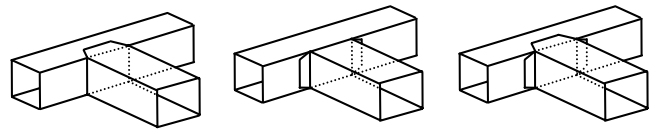

Figure 7 An example of joint library for 3-D beam based assemblies consisting of lap, butt and lap-butt.

\section{SCREW THEORY ${ }^{\dagger}$}

In Screw Theory, a screw is defined as a pair of a straight line (screw axis) in a 3D Cartesian space and a scalar (pitch). It is commonly represented by screw coordinates, a pair of two row vectors $\Sigma=\left(s ; \boldsymbol{s}_{0}\right)$ in 3D Cartesian coordinates, where $\boldsymbol{s}$ is a unit vector parallel to the screw axis and

$\boldsymbol{s}_{0}=\boldsymbol{r} \times \boldsymbol{s}+p \boldsymbol{s}$

In the equation, $r$ is the position vector of a point on the screw axis and $p$ is the pitch, which can be recovered using

\footnotetext{
${ }^{\dagger}$ The terminology and formalization in this section are summarized from Ball (1900), Hunt (1978), Roth (1984) and Konkar and Cutkosky (1995).
} 
$p=\frac{\mathbf{s} \cdot \mathbf{s}_{0}}{\mathbf{s} \cdot \mathbf{s}}$

A screw with an infinite pitch does not follow Equation (2), instead it is denoted by using zero vector for $\boldsymbol{s}$ and having $\boldsymbol{s}_{0}$ represent the unit vector parallel to the screw axis.

Two types of special screws, a twist and a wrench, are utilized in this paper. A twist is a screw representing a motion of a rigid body simultaneously rotating around and translating along an axis. Using screw coordinates, it is denoted as $\mathrm{T}=(\boldsymbol{\omega}, \boldsymbol{v})$, where $\boldsymbol{\omega}$ is the angular velocity and $\boldsymbol{v}$ is the linear velocity of a point on the body (or its extension) located at the origin of global reference frame. A wrench is a screw representing a force along and a moment around an axis exerted on a rigid body. Using screw coordinates, it is denoted as $\Omega=(\boldsymbol{f} ; \boldsymbol{m})$, where $\boldsymbol{f}$ is the force and $\boldsymbol{m}$ is the moment that a point on the body (or its extension) located at the origin of global reference frame should resist.

Two screws, $\Sigma_{1}=\left(\boldsymbol{s}_{1} ; \boldsymbol{s}_{01}\right)$ and $\Sigma_{2}=\left(\boldsymbol{s}_{2} ; \boldsymbol{s}_{02}\right)$, are reciprocal to each other, if and only if they satisfy:

$\boldsymbol{s}_{1} \cdot \boldsymbol{s}_{02}+\boldsymbol{s}_{01} \cdot \boldsymbol{s}_{2}=0$.

If a twist $\mathrm{T}$ is a reciprocal of wrench $\Omega$ (or vise versa), $\Omega$ does no "work" to a rigid body moving according to $\mathrm{T}$.

When a body can receive linear combinations of several screws (either twist or wrench), this set of screws are typically represented as a matrix where each screw in the set forms a row vector of the matrix. This matrix is called a screw matrix. As its row space is the screw space, the rank of a screw matrix is equal to the dimension of the screw space.

The function reciprocal(S) returns a screw matrix, of which row space includes all reciprocal screws to screws contained in $\mathbf{S}$. It can be obtained by exchanging the former three columns and the letter three columns of the null space of $\mathbf{S}$.

The union of screw matrices represents the sum of screw spaces and it can be obtained by simply "stacking" them on top of one another:

$$
\bigcup_{i=1}^{n} \mathbf{S}_{i}=\left(\begin{array}{c}
\mathbf{S}_{1} \\
\mathbf{S}_{2} \\
\vdots \\
\mathbf{S}_{n}
\end{array}\right)
$$

The intersection of screw matrices is the set of screws common to the screw matrices and can be computed through double reciprocals:

$$
\bigcap_{i=1}^{n} \mathbf{S}_{i}=\operatorname{reciprocal}\left(\bigcup_{i=1}^{n} \operatorname{reciprocal}\left(\mathbf{S}_{i}\right)\right)
$$

Since a twist and a wrench are also screws, the definitions of reciprocal, union, and intersection hold.

Woo and Freudenstein (1970) presents kinematic properties of various joint types in screw coordinates, which are borrowed to build twist matrices of beam joint types. (a)

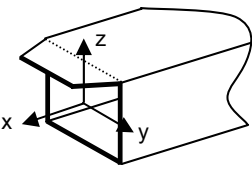

(b)

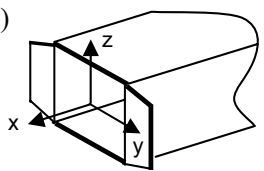

Figure 8 Lap (a) and lap-butt joint (b) of a beam based model and the local coordinate frames for twists.

Figure 8 (a) shows a typical lap joints found in beambased structures. When it is attached to another beam, the tab allows planar motion parallel to $x-y$ plane. Also, if we assume that the length of the tab is very small compared to the length of the beam, it can be treated as a line contact along $\mathrm{y}$-axis, thus allowing the rotation about y-axis. Thus, a lap joint at its local coordinate frame can be modeled as a twist matrix:

$\mathbf{T}_{\text {lap }}=\left(\begin{array}{llllll}0 & 1 & 0 & 0 & 0 & 0 \\ 0 & 0 & 1 & 0 & 0 & 0 \\ 0 & 0 & 0 & 1 & 0 & 0 \\ 0 & 0 & 0 & 0 & 1 & 0\end{array}\right)$

Similarly, a butt joint in Figure 8 (b) allows the motion parallel to y-z plane, can be modeled as:

$$
\mathbf{T}_{\text {butt }}=\left(\begin{array}{cccccc}
1 & 0 & 0 & 0 & 0 & 0 \\
0 & 0 & 0 & 0 & 1 & 0 \\
0 & 0 & 0 & 0 & 0 & 1
\end{array}\right)
$$

In twist matrices in equation (7) and (8), each row represents an independent motion, and each non-zero number represents rotation or translation along a corresponding axis $-\omega_{x}, \omega_{y}, \omega_{z}, v_{x}, v_{y}$ or $v_{z}$. For example, in the first row in Equation (7) has 1 at the second column, which means the lap joint allows rotational motion about $y$-axis. In the third row, it has 1 at the fourth column, meaning translation along the 
$\mathrm{x}$-axis. As these matrices are used only to give information on which DOFs are constrained for a joint type, amplitude of each twist (row) of these twist matrices, in this paper, does not have significant meaning.

Once the twist matrix is obtained for a joint type, the reciprocal wrench matrix can be computed as described above, and the wrench matrices corresponding to twist matrices in (7) and (8) are:

$$
\begin{aligned}
& \mathbf{W}_{\text {lap }}=\operatorname{reciprocal}\left(\mathbf{T}_{\text {lap }}\right)=\left(\begin{array}{llllll}
0 & 0 & 1 & 0 & 0 & 0 \\
0 & 0 & 0 & 1 & 0 & 0
\end{array}\right) \\
& \mathbf{W}_{\text {butt }}=\operatorname{reciprocal}\left(\mathbf{T}_{\text {but }}\right)=\left(\begin{array}{llllll}
1 & 0 & 0 & 0 & 0 & 0 \\
0 & 0 & 0 & 0 & 1 & 0 \\
0 & 0 & 0 & 0 & 0 & 1
\end{array}\right)
\end{aligned}
$$

Each non-zero number now represents force or moment along a corresponding axis $-f_{x}, f_{y}, f_{z}, m_{x}, m_{y}$ or $m_{z}$ - that the joint can constrain. For example, in the first row in Equation (9) has 1 at the third column, which means the lap joint can support a force along z-axis.

\section{3D ASSEMBLY SYNTHESIS}

\subsection{Binary decomposition}

The assembly synthesis algorithm in (Lee and Saitou, 2003a) adopted in this paper assumes every assembly step combines two subassemblies. Conversely, the algorithm decomposes a configuration into two (sub)configurations, by removing some connections, which is equivalent of finding a cut-set ${ }^{\ddagger}$ (Foulds, 1991) of the configuration graph. In the following, $C S_{d}$ and $K C_{d}$ denote the cut-set and the set of $\mathrm{KCs}$ broken by a decomposition $d$, respectively. For the decomposition shown in Figure 9, $C S_{d}=\{(1,2),(3$, 4) $\}$ and $K C_{d}=\{k c 1, k c 2\}$.

Any configuration $C_{a}=\left(M_{a}, E_{a}, A_{a}\right)$ decomposed to two subconfigurations $C_{b}=\left(M_{b}, E_{b}, A_{b}\right)$ and $C_{c}=\left(M_{c}\right.$, $\left.E_{c}, A_{c}\right)$, must satisfy the following conditions:

$M_{b} \neq \varnothing$ and $M_{c} \neq \varnothing$.

$\left(M_{a}, E_{a}\right),\left(M_{b}, E_{b}\right)$ and $\left(M_{c}, E_{c}\right)$ are connected.

$M_{a}=M_{b} \cup M_{c}$.

$M_{b} \cap M_{c}=\varnothing$.

The $1^{\text {st }}$ condition states subconfigurations should be nonempty. The $2^{\text {nd }}$ condition states the configurations

\footnotetext{
* In a configuration graph, edges representing KCs are not counted to a cut-set.
}

must be connected before and after decomposition. The $3^{\text {rd }}$ and $4^{\text {th }}$ conditions specify two subconfigurations do not share any members.

A joint is assigned to each connection broken by a binary decomposition, which can be represented as a mapping $\gamma_{d}: C S_{d} \mapsto J L$, where $J L$ is a joint library. With the joint assignment, a (binary) decomposition $d$ can be uniquely specified as $d=\left(M_{a}, \gamma_{d},\left(M_{b}, M_{c}\right)\right)$. See Figure 10 for an example. Note that a feasible joint type may depend on the local geometry at the joint location. For example, feasible joint types between two perpendicular beams would be different from that for two coaxial beams.

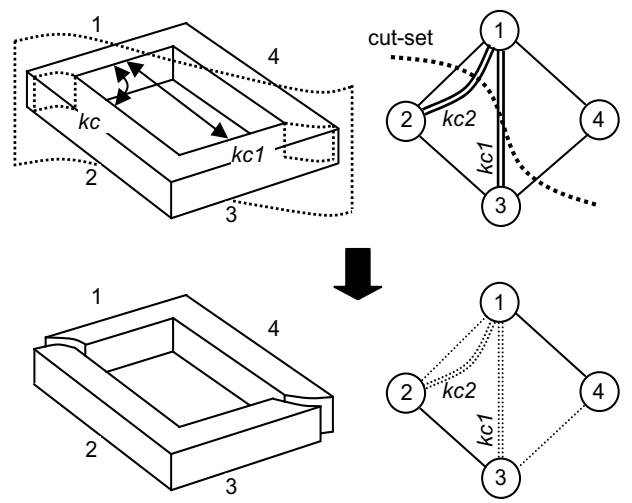

Figure 9 A binary decomposition in product geometry (left) and configuration graph (right).
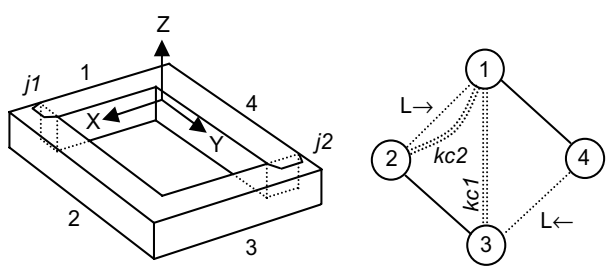

Figure 10 Joint types assigned to the subconfiguratins in Figure 9. The ' $\mathrm{L} \rightarrow$ ' represents a lap joint from a lower-index node to a higher-index node.

\subsection{The $1^{\text {st }}$ decomposition rule for in- process dimensional adjustability}

Let us consider how to assign appropriate joint types for those decompositions which have at least one broken KC. Recall Figure 3, which has a slip plane between parts 2 and 3 such that the $\mathrm{KC}$ can be delivered. The assembly sequence in Figure 3 (b) shows that it is desirable that a slip plane is provided 
at the very assembly operation where $\mathrm{KC}$ is realized, no matter how subassemblies are assembled before. This can be stated in the reverse course as follows: no matter how a subconfiguration is decomposed further, when KCs are broken by a decomposition, joints assigned to the cut-set, in combination, should allow motions compatible with the KCs. This statement has been refered to as the $1^{\text {st }}$ decomposition rule for in-process dimensional adjustability (Lee and Saitou, 2003a).

A $\mathrm{KC}$, in this paper, is assumed to be a critical dimension between parts only achieved by adjustment during assembly of the parts. Thus the dimension noted as a $\mathrm{KC}$ will be constrained by a fixture, according to which parts being assembled will be adjusted. In this context, KC constrains relative DOFs between two parts; hence it is natural to model a $\mathrm{KC}$ as a wrench matrix. The approach taking tolerance relations as constraints can be found in the area of computer-based tolerance modeling, and a recent study by $\mathrm{Wu}$ et. al. (2003) shows the number and the type of DOFs constrained for each tolerance relation in standard tolerance classes. In this paper, we consider only distance and angularity between lines (beams' axes). The distance between lines constrain only one translational DOF between two points where the $\mathrm{KC}$ is anchored, thus it is modeled as a wrench whose axis passes these points. The angularity between lines constrains only one rotational DOF between two lines and it is modeled as a wrench with infinite pitch whose axis is the vector product of the two lines' direction vectors.

The $1^{\text {st }}$ decomposition rule for in-process dimensional adjustability, in other words, states that the DOFs constrained by KCs should not be constrained by the joints, thus avoiding conflicts. Once wrench matrices are associated to joints and $\mathrm{KCs}$ broken by a decomposition, this rule can be stated in the screw theory's terminology: for a decomposition, the wrenches representing joints and $\mathrm{KCs}$ should not constrain the same DOF, thus satisfying:

$$
\left(\bigcup_{e \in C S_{d}} \mathbf{W}_{\gamma_{d}(e)}\right) \cap\left(\bigcup_{a \in K C_{d}} \mathbf{W}_{a}\right)=\mathbf{0}
$$

Since the rank of the intersection of the joint and KC matrices is zero as shown in equation (11), by the theorem from linear algebra, it is obvious that the rank in equation (12) is merely summation of ranks of joint and $\mathrm{KC}$ matrices:

$$
\begin{aligned}
& \operatorname{rank}\left(\left(\bigcup_{e \in C S_{d}} \mathbf{W}_{\gamma_{d}(e)}\right) \cup\left(\bigcup_{a \in K C_{d}} \mathbf{W}_{a}\right)\right) \\
& \quad=\operatorname{rank}\left(\bigcup_{e \in C S_{d}} \mathbf{W}_{\gamma_{d}(e)}\right)+\operatorname{rank}\left(\bigcup_{a \in K C_{d}} \mathbf{W}_{a}\right)
\end{aligned}
$$

Furthermore, as the proper constraint design in its rigorous definition avoids under-constraint as well as over-constraint, the combined constraints from joints and KCs should cover six DOFs, such that no DOF could be left unconstrained when two parts are being assembled. In other words, the dimension of the combined wrench space, i.e., the rank of the union of joint and $\mathrm{KC}$ wrench matrices, should be equal to six. Combined with equation (12), we can now conclude the $1^{\text {st }}$ rule of decomposition for in-process dimensional adjustability with:

$$
\begin{aligned}
& \operatorname{rank}\left(\left(\bigcup_{e \in C S_{d}} \mathbf{W}_{\gamma_{d}(e)}\right) \cup\left(\bigcup_{a \in K C_{d}} \mathbf{W}_{a}\right)\right) \\
& =\operatorname{rank}\left(\bigcup_{e \in C S_{d}} \mathbf{W}_{\gamma_{d}(e)}\right)+\operatorname{rank}\left(\bigcup_{a \in K C_{d}} \mathbf{W}_{a}\right)=6 .
\end{aligned}
$$

Consider the product geometry decomposed in Figure 9 and joint assignment shown in Figure 10, which has two lap joints, $j 1$ and $j 2$ for edges cut by the decomposition. Suppose the location of $j 1$ and $j 2$ in global reference frame X-Y-Z are $(3,0,0)$ and $(0$, $4,0)$. Then, based on the local coordinate frame of lap joint shown in Figure 8 and orientation of $j 1$ and $j 2, \mathbf{W}_{\text {lap }}$ (Equation (9)) can be transformed to $j 1$ and $j 2$ in global reference frame. Then the union of joint wrench matrices can be computed:

$$
\bigcup_{e \in C S_{d}} \mathbf{W}_{\gamma_{d}(e)}=\mathbf{W}_{j l} \cup \mathbf{W}_{j 2} \sim\left(\begin{array}{cccccc}
0 & 0 & 1 & 0 & 0 & 0 \\
0 & 0 & 0 & 1 & 0 & 0 \\
0 & 0 & 0 & 0 & 1 & 0
\end{array}\right) \S(14)
$$

The wrench matrix in (14) has 1 at the third, fourth and fifth column, meaning that it supports force along Z-axis, moments about $\mathrm{X}$ and $\mathrm{Y}$ axis, respectively. On the other hand, the decomposition in Figure 9 has broken two KCs, $k c 1$ and $k c 2$. The union of these $\mathrm{KCs}$ is:

$$
\bigcup_{a \in K C_{d}} \mathbf{W}_{a}=\mathbf{W}_{k c 1} \cup \mathbf{W}_{k c 2}=\left(\begin{array}{cccccc}
0 & 1 & 0 & 0 & 0 & 1.5 \\
0 & 0 & 0 & 0 & 0 & 1
\end{array}\right) \text {. }
$$

Note that $\mathbf{W}_{k c l}$ (upper row) represents the distance $\mathrm{KC}$ between member 1 and 3 (translation along Yaxis) and $\mathbf{W}_{k c 2}$ (lower row) represents the angularity $\mathrm{KC}$ between member 1 and 2 (rotation about $\mathrm{Z}$-axis).

\footnotetext{
$\S$ The result has been reduced to the Row Reduced Echelon Form for easy interpretation.
} 
The union of the joint twist matrix (Equation (14)) and $\mathrm{KC}$ twist matrix (Equation (15)) is:

$$
\left(\bigcup_{e \in C S_{d}} \mathbf{W}_{\gamma_{d}(e)}\right) \cup\left(\bigcup_{a \in K C_{d}} \mathbf{W}_{a}\right) \sim\left(\begin{array}{cccccc}
0 & 1 & 0 & 0 & 0 & 0 \\
0 & 0 & 1 & 0 & 0 & 0 \\
0 & 0 & 0 & 1 & 0 & 0 \\
0 & 0 & 0 & 0 & 1 & 0 \\
0 & 0 & 0 & 0 & 0 & 1
\end{array}\right) .
$$

It shows that the parts are constrained in $\mathrm{X}$-axis neither by joints nor by KCs. Although it does not satisfy Equation (13), it does satisfy Equation (12), which implies at least that there is no conflict between joints and KCs. As this decomposition does not satisfy Equation (13), the assembly synthesis process will discard it.

\subsection{The 2nd decomposition rule for in- process dimensional adjustability}

As discussed in Figure 4, when multiple KCs in the same direction are realized at an assembly step, the adjustment of one $\mathrm{KC}$ will affect the dimension of the other KCs. Viewing KCs as constraints, this happens when two or more KCs constrain the same DOF of a subassembly at an assembly step. However, for complex assemblies, detecting over-constrained tolerance relationship is not straightforward from the engineering drawings because tolerances are specified on parts, not subassemblies, which are defined by assembly sequences. Therefore, a clumsy assembly planning might cause a subassembly's DOF to be constrained by several KCs. In order to avoid this situation, one should plan assembly steps such that, in every assembly step, subassemblies being assembled are free of over-constraining KCs.

Accordingly, the $2^{\text {nd }}$ decomposition rule for inprocess dimensional adjustability in (Lee and Saitou, 2003a) states a decomposition can break only KCs independent to each other ${ }^{* *}$. In other words, $\mathrm{KCs}$ broken by a decomposition, i.e., the $\mathrm{KCs}$ in $K C_{d}$, should not constrain the same DOF more than once. In such cases, the intersection of the wrench matrix corresponding to any subset of $K C_{d}$ and the wrench matrix of its complement set must result in the zero matrix:

$$
\forall K \subseteq K C_{d},\left(\bigcup_{a \in K} \mathbf{W}_{a}\right) \cap\left(\bigcup_{a \in K C_{d} \backslash K} \mathbf{W}_{a}\right)=\mathbf{0}
$$

\footnotetext{
${ }^{* *}$ In $2 \mathrm{D}$ cases in the previous works, only $\mathrm{KCs}$ perpendicular to each other were allowed to be stricter.
}

, which is also equivalent to:

$\operatorname{rank}\left(\bigcup_{a \in K C_{d}} \mathbf{W}_{a}\right)=\sum_{a \in K C_{d}} \operatorname{rank}\left(\mathbf{W}_{a}\right)$.

The two KCs shown in Figure 9, each with single wrench make the KC matrix of rank 2 as shown in Equation (15), thus satisfying Equation (18).

\subsection{The decomposition rule for in- process proper constraint}

In Figure 5, it has been shown that joints should be perpendicular to each other to have subassemblies being assembled properly constrained. Similarly to drawing the first and second decomposition rule for in-process dimensional adjustment, this assembly rule has been inversed to the decomposition rule for non-forced fit in our previous work (Lee, B. and Saitou, K., 2003a), which allows only mutually perpendicular joints to be broken by a decomposition. This rule is simplified and limited to twodimensional space assuming over-constraints in rotation are minimal.

The idea of this rule is that there should be no overconstraint at each assembly step, hence the decomposition rule (renamed as the decomposition rule for in-process proper constraint) should not allow any combination of joints yielding overconstraint of parts. In other words, joints placed for connections broken by a decomposition, i.e., the joints corresponding to $C S_{d}$, should not constrain the same DOF more than once. Except that joints serve as constraints, instead of $\mathrm{KCs}$, this rule is identical to the $2^{\text {nd }}$ rule of in-process dimensional adjustability, thus satisfying:

$$
\forall C \subseteq C S_{d},\left(\bigcup_{e \in C} \mathbf{W}_{\gamma_{d}(e)}\right) \cap\left(\bigcup_{e \in C S_{d} \backslash C} \mathbf{W}_{\gamma_{d}(e)}\right)=\mathbf{0}
$$

, which is also equivalent to:

$$
\operatorname{rank}\left(\bigcup_{e \in C S_{d}} \mathbf{W}_{\gamma_{d}(e)}\right)=\sum_{e \in C S_{d}} \operatorname{rank}\left(\mathbf{W}_{\gamma_{d}(e)}\right) .
$$

For the decomposition depicted in Figure 10, each of the two joints $j 1$ and $j 2$ has rank 2 (Equation (9)). However, the union of corresponding wrench matrices has rank 3, which does not satisfy Equation (20). In order to check what DOFs are overconstrained, we can intersect the wrench matrices:

$$
\begin{aligned}
\mathbf{W}_{j 1} \cap \mathbf{W}_{j 2} & =\operatorname{recip}\left(\operatorname{recip}\left(\mathbf{W}_{j 1}\right) \bigcup \operatorname{recip}\left(\mathbf{W}_{j 2}\right)\right) \\
& =\operatorname{recip}\left(\mathbf{T}_{j 1} \cup \mathbf{T}_{j 2}\right) \\
& =\left[\begin{array}{llllll}
0 & 0 & 1 & 4 & -3 & 0
\end{array}\right] \neq \mathbf{O}
\end{aligned}
$$


The results states that the joints over-constrain the translational DOF along Z-axis, which yields locked moment about $\mathrm{X}$-axis with unit of 4 and moment about Y-axis with unit of -3 . It occurs because $j 1$ itself constrains parts both in translation along $\mathrm{Z}$-axis and the moment about $\mathrm{X}$-axis at the same time $j 2$ combined with $j 1$ constrain the moment about $\mathrm{X}$-axis again. And $j 2$ and $j 1$ cooperate in the same way to result in the locked moment about $\mathrm{Y}$-axis.

\subsection{Unified decomposition rule for in- process proper constraint}

According to Equation (18), the set of KCs related to a decomposition should be linearly independent. Similarly, the set of joints assigned for broken connections should be linearly independent according to Equation (20). Further, as these sets should be linearly independent to each other by Equation (13), these three equations in combination requires the independency of constraints, regardless of $\mathrm{KC}$ or joint, and full rank when unionized. Thus, combining Equation (13), (18) and (20), we can unify three decomposition rules into:

$$
\begin{aligned}
& \operatorname{rank}\left(\left(\bigcup_{e \in C S_{d}} \mathbf{W}_{\gamma_{d}(e)}\right) \cup\left(\bigcup_{a \in K C_{d}} \mathbf{W}_{a}\right)\right) \\
& =\sum_{e \in C S_{d}} \operatorname{rank}\left(\mathbf{W}_{\gamma_{d}(e)}\right)+\sum_{a \in K C_{d}} \operatorname{rank}\left(\mathbf{W}_{a}\right)=6 .
\end{aligned}
$$

Finally, a predicate of a decomposition $d=\left(M_{a}, \gamma_{d}\right.$, $\left(M_{b}, M_{c}\right)$ ) for complying the all three rules is given as de: $2^{M_{0}} \times\left(2^{E_{0}} \mapsto J L\right) \times\left(2^{M_{0}} \times 2^{M_{0}}\right) \mapsto\{$ true, false $\} \quad$, where $\operatorname{de}\left(M_{a}, \gamma_{d},\left(M_{b}, M_{c}\right)\right)$ is true if and only if Equation (22) is satisfied. However, it is often the case that a under-constraints are unavoidable during assembly synthesis due to the limited choice of joints, Equation (22) may be relaxed to abandon the full rank.

\subsection{Part manufacturability}

The decomposition stops when the resulting subconfigurations become manufacturable by a chosen manufacturing process. In the following case study on frame structures, components are assumed to be extruded and bent. Therefore, a predicate of a configuration $M_{a}$ for stopping decomposition is given as stop_de: $2^{M_{0}} \mapsto\{$ true, false $\}$, where stop_de $\left(M_{a}\right)$ is false (i.e.. decomposition continues) if and only if any of the following conditions are satisfied:

- $M_{a}$ has a KC (KCs can not be achieved by the tolerances of extrusion and bending).
- $M_{a}$ has a closed loop (cannot extrude such parts).

- $M_{a}$ has a connection point where three or more members meet (cannot extrude such parts).

- $\quad M_{a}$ has members lie on more than one plane (difficult to handle/fixture)

The product geometry shown in Figure 6 has two $\mathrm{KCs}$ and a closed loop thus stop_de returns false, subject to further decomposition.

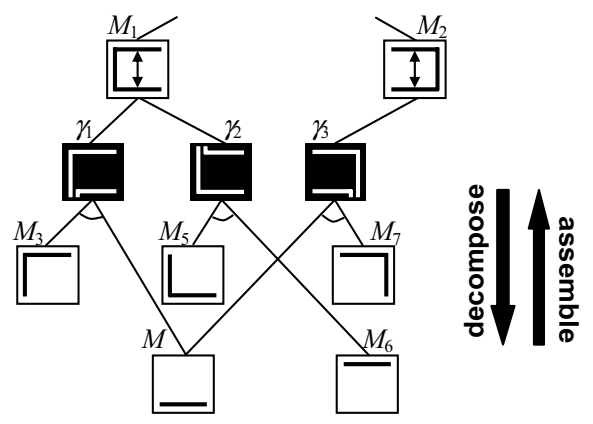

Figure 11 A partial AND/OR graph of the 2-D rectangular box in Figure 1.

\subsection{AND/OR graph of assembly synthesis}

Figure 11 shows a partial AND/OR graph of assembly synthesis (Lee and Saitou, 2003a) for the 2D rectangular box shown in Figure 1. Each node in white background contains a subset of members $\left(M_{a}\right.$ $\subseteq M_{0}$ ) and each node in black background contains joint assignment $\gamma_{i}: C S_{i} \mapsto J L$. A set of three lines which connects a configuration $M_{a}$, joint assignment $\gamma_{i}$, and two subconfigurations $\left(M_{b}, M_{c}\right)$ is a hyperedge, represented as $\left(M_{a}, \gamma_{i},\left(M_{b}, M_{c}\right)\right)$ which is also the representation of a decomposition defined earlier. The AND/OR graph of assembly synthesis is then represented as a triple:

$A O=(S, J, F)$

where $S$ is a set of nodes representing configurations, $J$ is a set of nodes representing joint assignments, and $F$ is a set of hyper-edges $\left(M_{a}, \gamma_{i},\left(M_{b}, M_{c}\right)\right)$ satisfying the following necessary conditions.

1. stop_de $\left(M_{a}\right)=$ false.

2. $\operatorname{de}\left(M_{a}, \gamma_{i},\left(M_{b}, M_{c}\right)\right)=$ true.

Then $A O=(S, J, F)$ is recursively defined as:

1. If stop_de $\left(M_{0}\right)=$ false, $M_{0} \in S$. 
2. For $\forall M_{a} \in S$, if $\exists \gamma_{i}, M_{b}, M_{c}$ such that $f=\left(M_{a}, \gamma_{i}\right.$, $\left(M_{b}, M_{c}\right)$ ) satisfies necessary conditions (19), then $\gamma_{i} \in J, M_{b}, M_{c} \in S$ and $f \in F$.

3. No element is in $S, J$ and $F$, unless it can be obtained by using rules 1 and 2 .

The recursive definition in Equation (25) can be easily transformed to an algorithm build_AO that generates $A O$ from initial configuration $C_{0}=\left(M_{0}, E_{0}\right.$, $\left.A_{0}\right)$ and joint library $J L$ by recursively decomposing a configuration into two subconfigurations (Lee and Saitou, 2003a), whose details are omitted due to space limitation. Using stop_de and de as defined earlier, one can run build_AO with any $3 \mathrm{D}$ configurations to enumerate all possible assemblies (decompositions and joint assignments) and accompanying assembly sequence that satisfy the rules for in-process dimensional adjustability and proper part constraint.

\section{CASE STUDY}

A frame structure in Figure 12 is decomposed using the joint types in Figure 13. Since the initial attempt yields no assembly synthesis without underconstraints, the Equation (22) is relaxed to allow under-constraints.

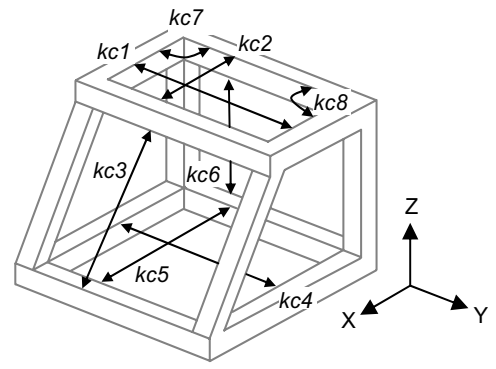

Figure 12 A frame structure with eight KCs.
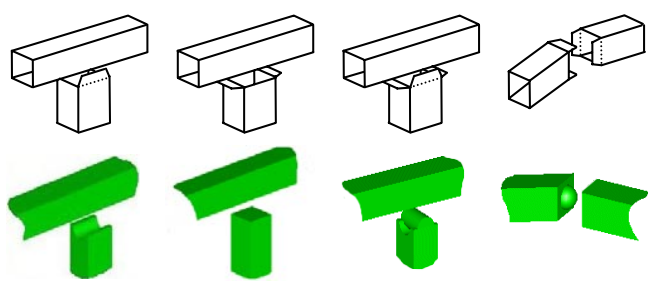

Figure 13 (top) joint types for frame sturcture, and (bottom) their graphical representation.

The relaxed rule produced the AND/OR graph of assembly synthesis with 112 feasible decompositions with 73 feasible subassemblies. Due to the space limit, we have extracted a part of the AND/OR graph containing the assembly designs with minimum number of parts and under-constrains. The extracted graph has 26 feasible decompositions with 28 feasible subassemblies, a half of which is depicted in Figure 14 as the product geometry is symmetrical about its XZ-plane. In Figure 14, white nodes are parts and grey nodes are subassemblies. Joint assignments are represented as black nodes with numbers, which represent the number of underconstraints related to the assembly step. The number of parts and the number of under-constraints do not exhibit any trade-off in this example, since in general assemblies with fewer parts have less chance to be under-constrained. All assemblies shown in Figure 14 have 7 parts and 2 under-constraints. There are no assemblies with fewer parts or under-constraints.

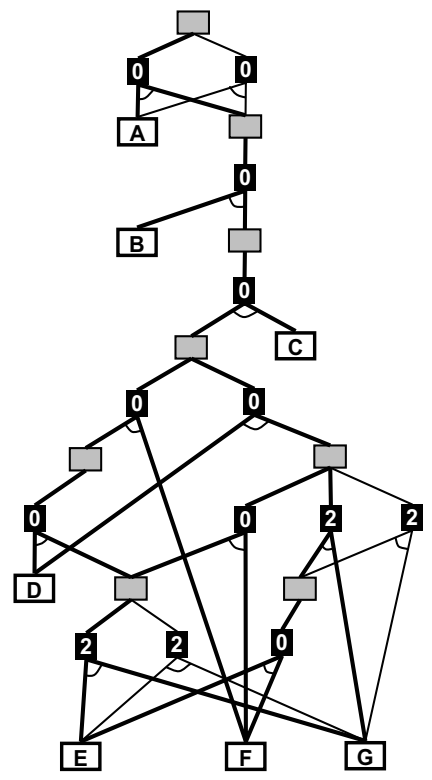

Figure 14 Half of optimal AND/OR graph of assembly synthesis for the product geometry in Figure 12.

Figure 15 shows all assembly designs in Figure 14. While the AND/OR graph has 12 trees, Figure 15 shows only one way of part decomposition with 4 variations in joint assignments, which is because there are three different assembly sequences for each assembly design in Figure 15. For example, the assembly design at top left in Figure 15 has three 
assembly sequences, which are thick-lined in Figure 14. One of these assembly sequences is as follows:

1. Assemble $\mathrm{E}$ and $\mathrm{G}$ to achieve $k c 2$ and $k c 8$. Underconstrained in Y-translation and Y-rotation.

2. Assemble D and E-G to achieve $k c l$ and $k c 7$. Properly constrained.

3. Assemble F and D-E-G to achieve $k c 3$. Properly constrained.

4. Assemble $\mathrm{C}$ and D-E-F-G without achieving any KC. Properly constrained.

5. Assemble B and C-D-E-F-G to achieve $k c 5$ and $k c 6$. Properly constrained.

6. Assemble A and B-C-D-E-F-G to achieve $k c 4$. Properly constrained.
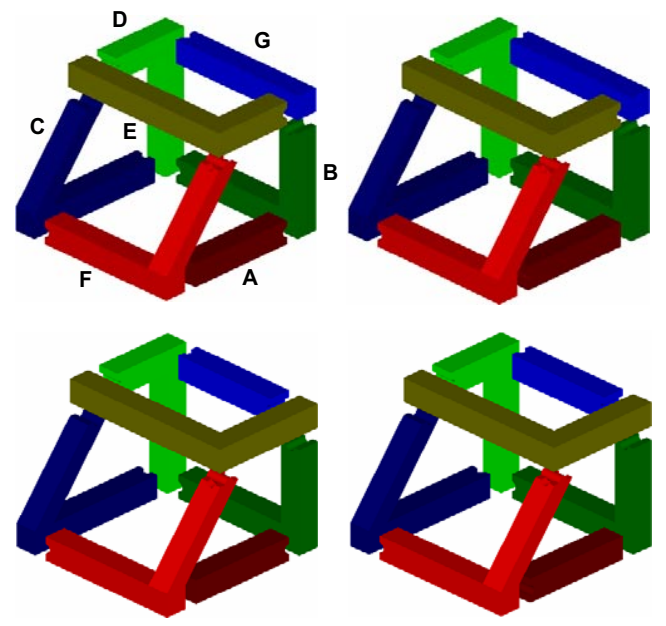

Figure 15 All assembly designs existing in the optimal AND/OR graph shown in Figure 14, where corresponding assembly sequences can be found.

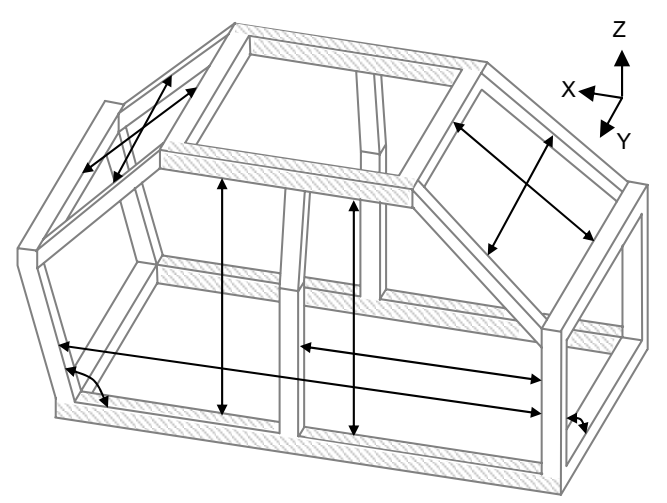

Figure 16 Passenger area of an automotive space frame.

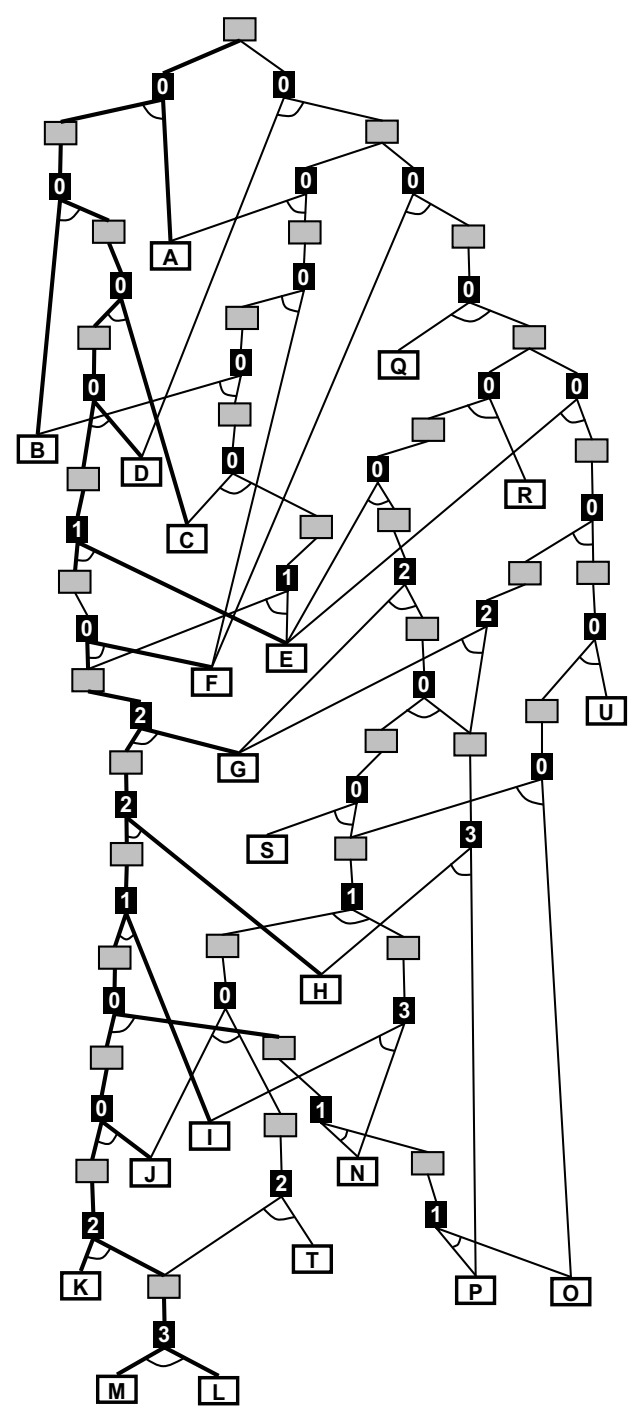

Figure $17 \mathrm{An}$ optimal assembly synthesized for the automotive space frame in Figure 16.

Figure 16 shows the passenger area of an automotive space frame with $18 \mathrm{KCs}$ (KCs at the far side of X-Z plane are symmetrical and omitted). Each horizontal beam at side (shaded area) has two members, however, we have not allowed them to part, in other to reflect practices and reduce the size of solution. With the same relaxed rule permitting underconstraints, the AND/OR graph contains 12420 feasible decompositions with 1492 feasible subassemblies. Among assemblies without any part 
failing the manufacturability criteria, the minimum number of parts is 15 and the minimum number of under-constrains is 13 . The optimal solution graph in terms of the number of parts and under-constraints with even weights contains 293 feasible
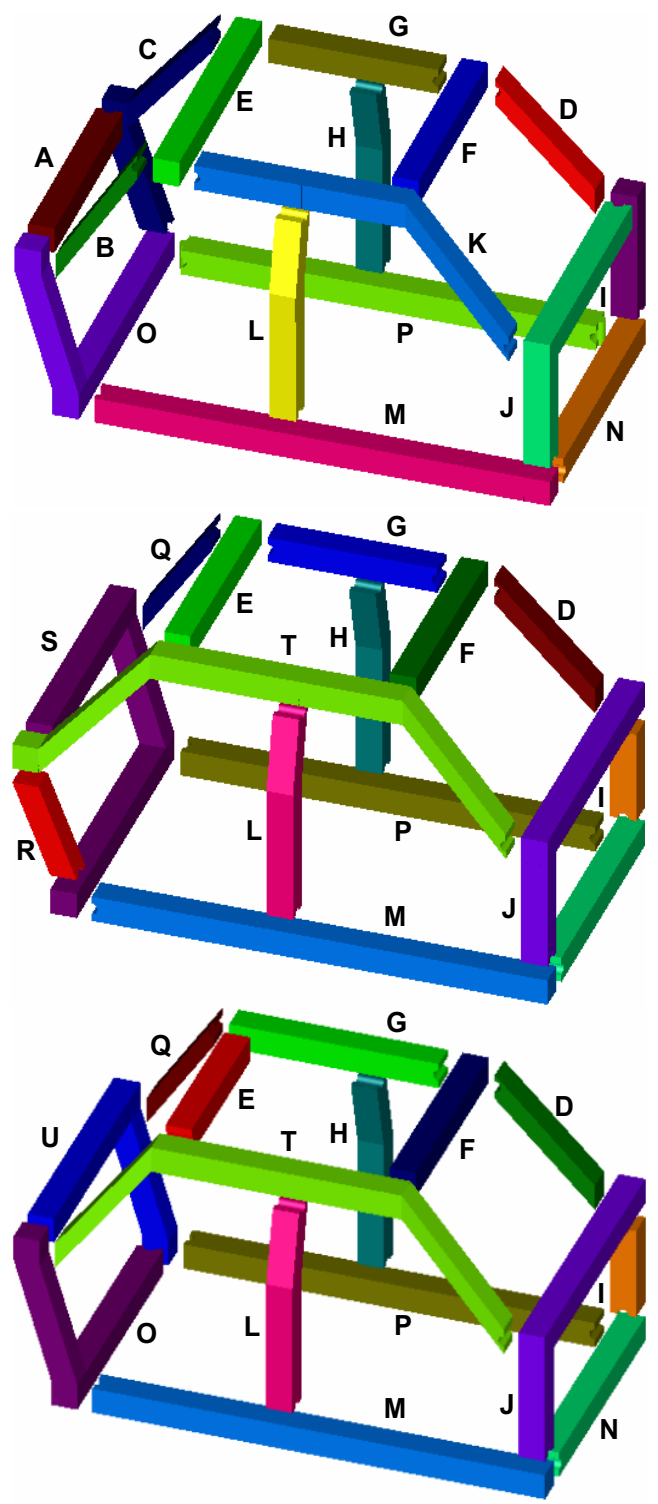

Figure 18 A partial optimal AND/OR graph of assembly synthesis for the automotive space frame in Figure 16. decompositions with 87 feasible subassemblies, in which each synthesized assembly consists of either 16 parts with 13 under-constraints or 15 parts with 14 under-constraints. Three of these optimal assembly designs are shown in Figure 18 and the corresponding AND/OR graph of assembly synthesis is shown in Figure 17. The first assembly design consists of 16 parts and has two different assembly sequences in the partial optimal solution graph, which yield 13 under-constraints during assembly steps. The other designs have 15 parts and each has one assembly sequence in the graph, yielding 14 under-constraints.

\section{SUMMARY AND DISCUSSION}

This paper presented a 3D assembly synthesis method for in-process dimensional adjustability and proper part constraint based on Screw Theory. While this paper focuses only on in-process adjustability and proper part constraints and suggest ideal designs for these criteria, other criteria, such as part symmetry and structural stiffness, also drive assembly synthesis in practice. The present method, therefore, would most effectively be integrated in the design process if it is applied to subassemblies of a product first decomposed by a human designer based on the current practice.

\section{ACKNOWLEDGMENTS}

This work has been supported by the National Science Foundation with a CAREER Award (DMI9984606) and Toyota Motor Company. Any opinions, findings, and conclusions or recommendations expressed in this material are those of the authors and do not necessarily reflect the views of the National Science Foundation.

\section{REFERENCES}

Adams, J. D. and Whitney, D. E., (1999), “Application of screw theory to constraint analysis of assemblies of rigid parts", Proceedings of the 1999 IEEE International Symposium on Assembly and Task Planning, Porto, Portugal, July 1999, pp. 69-74.

Ball, R. S., (1900), A Treatise on the Theory of Screws, Cambridge University Press.

Blanding, D. L., (1999), Exact Constraint: Machine Design Using Kinematic Principles, ASME Press, New York.

Downey, K, Parkinson, A. R and Chase, K. W., (2002), "Smart assemblies for robust design: a progress report," Proceedings of the 2002 ASME Design 
Engineering Technical Conferences and Computers and Information in Engineering Conference, Montreal, Canada, Sep 29-Oct 2, Paper no. DETC2002/DAC34135 .

Foulds, L. R., (1991), Graph Theory Applications, Springer-Verlag, New York, New York.

Homem de Mello, L. S. and Sanderson, A. C., (1990), "AND/OR graph representation of assembly plans," IEEE Transactions on Robotics and Automation, vol. 6, no. 2, pp. 188-199.

Hunt, K. H., (1978), Kinematic Geometry of Mechanisms, Oxford University Press.

Kamm, L. J., (1990), Designing Cost-Effective Mechanisms, McGraw-Hill.

Konkar, R. and Cutkosky, M., (1995), "Incremental kinematic analysis of mechanisms", Journal of Mechanical Design, vol. 117, pp. 589-596.

Kriegel, J. M., (1995), "Exact constraint design," Mechanical Engineering, vol. 117, no. 5, pp. 88-90.

Lee, B. and Saitou, K. (2003a), "Decomposition-based assembly synthesis for in-process dimensional adjustability," ASME Journal of Mechanical Design, vol. 125 , no. 3, pp. 464-473.

Lee, B. and Saitou, K. (2003b), “Assembly synthesis with subassembly partitioning for optimal in-process dimensional adjustability," Proceedings of the 2003 ASME Design Engineering Technical Conferences, Chicago, Illinois, Sep 2-6, Paper no. DETC2003/DAC48730.

Roth, B., (1983), "Screws, motors, and wrenches that can not be bought in a hardware store," Robotics Research, The first symposium, MIT Press, Cambridge, MA, pp. 679-735.

Sugimoto, K. and Duffy, J., (1982), “Application of linear algebra to screw systems," Mechanism and Machine Theory, vol. 17, no. 1, pp. 73-83.

Waldron, K. J., (1966), "The constraint analysis of mechanisms," Journal of Mechanisms, vol. 1, no. 2, pp. 101-114.

Whitehead, T. N., (1954), The Design and Use of Instruments and Accurate Mechanism, Dover Publications, New York, New York.

Whitney, D. E., Mantripragada, R., Adams, J. D., and Rhee, S. J., (1999), "Designing assemblies," Research in Engineering Design, vol. 11, pp. 229-253.

Woo, L. and Freudenstein, F., (1970), “Application of line geometry to theoretical kinematics and the kinematic analysis of mechanical systems," Journal of Mechanisms, vol. 5, pp. 417-460.
Wu, Y., Shah, J. J. and Davison, J. K., (2003), "Computer modeling of geometric variations in mechanical parts and assemblies," ASME Journal of Computing and Information Science in Engineering, vol. 3, no.1, pp. 54-63. 